Effects of alloy potential fluctuations in InGaN epitaxial films

This article has been downloaded from IOPscience. Please scroll down to see the full text article.

1999 Semicond. Sci. Technol. 14406

(http://iopscience.iop.org/0268-1242/14/5/006)

The Table of Contents and more related content is available

Download details:

IP Address: 140.112.113.225

The article was downloaded on 26/06/2009 at 09:38

Please note that terms and conditions apply. 


\title{
Effects of alloy potential fluctuations in InGaN epitaxial films
}

\section{T Y Lin, J C Fan and Y F Chen}

Department of Physics, National Taiwan University, Taipei, Taiwan, Republic of China

Received 27 October 1998, accepted for publication 2 February 1999

\begin{abstract}
Results of photoluminescence and photoconductivity measurements in $\operatorname{In}_{x} \mathrm{Ga}_{1-x} \mathrm{~N}$ epitaxial films are presented. The photoluminescence peak energy and intensity show several anomalous behaviours. The peak energy changes with temperature exhibiting an inverted $\mathrm{S}$-shape dependence, where it decreases, then increases with increasing temperature in the range $40-100 \mathrm{~K}$ and finally decreases with increasing temperature. The intensity shows a temperature dependence similar to that of amorphous semiconductors and disordered superlattices. A blue shift of the photoluminescence energy with increasing excitation intensity is observed. A large Stokes shift between the photoluminescence peak position and the band edge transition energy is found; it decreases with decreasing indium content. A persistent photoconductivity effect has been detected up to room temperature with a stretched-exponential function for its decay rate. All these observations can be explained in a consistent way by alloy potential fluctuations, and these clearly indicate the existence of compositional fluctuations. These two related effects thus appear to constitute the mechanism for the widely observed localized excitons in InGaN-based devices.
\end{abstract}

\section{Introduction}

Recently, InGaN alloys have been attracting much attention as potential materials for the fabrication of short-wavelength light-emitting diodes and injection lasers because of the advantage of tuning ability of the alloy bandgap. The growth of high-quality InGaN films with a high indium composition, however, is not well developed because of the compositionally unstable nature of the alloy or the deposition of indium droplets. These materials were found to show broad lines in x-ray diffraction and optical spectroscopy indicating layer alloy fluctuations [1]. Such fluctuations have also been directly observed by scanning transmission electron microscopy [2]. Nevertheless, the surprising evidence is that the InGaN alloys exhibit intense electroluminescence (EL) or photoluminescence (PL) intensity in spite of their large threading dislocation densities. A detailed understanding of the physical origins of the emission lines in these materials is very important for the improvement of material quality as well as the design of optical devices.

It was demonstrated previously that the PL emissions in InGaN epilayers result primarily from localized exciton recombination $[3,4]$. The localized excitons were recently found to play an important role in the lasing process of InGaN-based single and multiple quantum wells (QWs) at room temperature (RT) [5,6]. In general, the depth of the trap centre was found to increase with the indium component, $x$, and to be much larger than the thermal energy at RT both in epifilms and in QW structures with $x \leqslant 0.2$. However, the origin of the trap centre has not been clarified yet.
In this paper, we report several peculiar behaviours of optical properties observed in $\operatorname{In}_{1-x} \mathrm{Ga}_{x} \mathrm{~N}$ thin films with different indium concentrations, which include the dependence of PL spectra on temperature and excitation intensity and the existence of a large Stokes shift between the PL peak position and the band edge transition energy, as well as the observation of persistent photoconductivity (PPC). All the anomalous behaviours can be explained by the effect of alloy potential fluctuations (APFs) in a unified way. The presence of the random local alloy fluctuations in composition and therefore potential in the alloy layers usually results in the localized band tail states. Based on the existence of the localized tail states, all our measurements can be clearly understood. Our results thus firmly establish the existence of compositional fluctuations in InGaN epilayers and provide the evidence showing that APFs can significantly influence the properties of materials.

\section{Experiment}

The samples used in this study were nominally undoped single-crystal epilayers grown by low-pressure metal-organic chemical vapour deposition. Before the deposition of InGaN alloys, a $20 \mathrm{~nm}$ thick $\mathrm{GaN}$ buffer layer was grown on a $c$-plane sapphire substrate at a temperature of $1050^{\circ} \mathrm{C}$. The alloy layers were deposited at a temperature around $800^{\circ} \mathrm{C}$. The thickness of the $\operatorname{In}_{x} \mathrm{Ga}_{1-x} \mathrm{~N}$ epilayers was typically around $1000 \AA$. A Hall-effect measurement was performed at RT by the van der Pauw method; the result shows that the background electron concentration of the 
InGaN films is about $10^{18} \mathrm{~cm}^{-3}$ at RT. The sample was attached to a sample holder mounted onto the cold finger of a closed-cycle He refrigerator with care to ensure good thermal contact yet electrical isolation. The measurements were carried out over a temperature range from 10 to $300 \mathrm{~K}$. All the data obtained under different conditions were taken in such a way that the system was always allowed to relax to equilibrium. This was to ensure that the data obtained had the same initial condition. For the photoconductivity (PC) measurements, ohmic contacts were formed by depositing indium drops to the four corners of the sample and annealing the sample at $400{ }^{\circ} \mathrm{C}$ for $10 \mathrm{~s}$. A tungsten lamp dispersed by a monochromator was used as the photoexcitation light source. A constant direct current was applied and the conductivity was measured by a Keithley 236 source measure unit. Details of PPC measurement procedure were similar to those described previously [7]. For the PL measurements, the sample was excited by an $\mathrm{He}-\mathrm{Cd}$ laser working at $325 \mathrm{~nm}$, dispersed by a Spex $1 \mathrm{~m}$ double-grating spectrometer, and the spectrum was detected by a cooled GaAs photomultiplier. A detailed description of the setup of the PL measurements has been published elsewhere [8].

\section{Results and discussion}

A typical PL spectrum of the undoped InGaN epifilm sample taken at $10 \mathrm{~K}$ is shown in figure 1 . The spectrum is dominated by a single emission band with a peak centred at $2.79 \mathrm{eV}$ and there is no observable deep level emission. According to a previous report [9], the peak position centred at $2.79 \mathrm{eV}$ corresponds to $30 \%$ indium content. The full width at halfmaximum of the PL spectrum is about $200 \mathrm{meV}$ which is comparable with the reported data [9]. To investigate further the alloy properties of the InGaN epilayer, we measured the dependence of the PL spectra on temperature and excitation intensity. Figure 2 shows the integrated PL emission intensity as a function of temperature. It appears that the data cannot be fitted by an Arrhenius plot. Instead, the relationship is similar to that used for amorphous semiconductors and disordered superlattices $[10,11]$,

$$
I_{P L}=I_{0} /\left[1+A \exp \left(T / T_{0}\right)\right]
$$

where $I_{P L}$ is the PL intensity, $T$ the temperature and $T_{0}$ the characteristic temperature corresponding to the energy depth from radiative state to nonradiative state. $A$ is the tunnelling factor and $I_{0}$ is the luminescence intensity at the low-temperature limit. Equation (1) is valid if localized bandtail states exist [10-12]. Therefore, it seems reasonable to infer that the PL signal in the InGaN epilayer comes from localized states. Recently, the existence of localized excitons has also been suggested in InGaN epifilms and QW structures from PL, EL, PL excitation and time-resolved PL measurements [3-6]. The origin of the localized states could be due to the existence of APF in the InGaN films, because APF is one of the most important aspects that belong to the inherent nature of substitutional semiconductor alloys. It has been shown in many semiconductor alloys that APF can greatly change the electrical and optical properties of a material, such as carrier mobility [13], PL profile [14], Raman

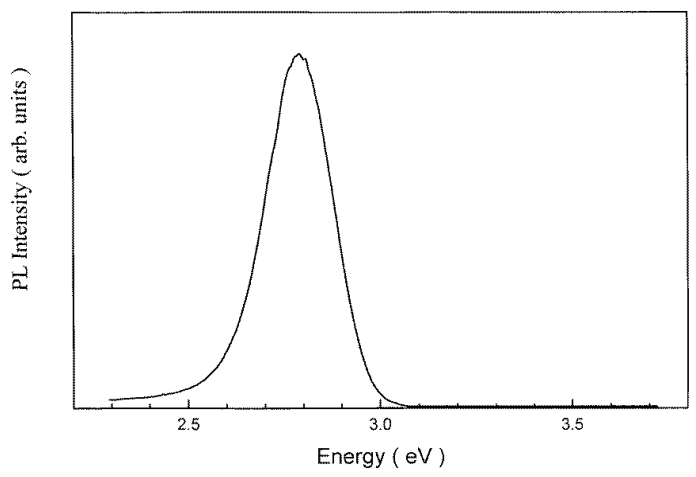

Figure 1. PL spectrum of an undoped $\operatorname{In}_{0.30} \mathrm{Ga}_{0.70} \mathrm{~N}$ epifilm sample taken at $10 \mathrm{~K}$.

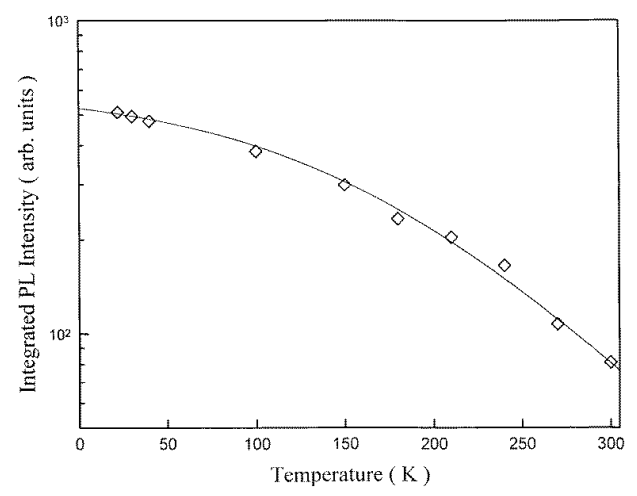

Figure 2. Temperature dependence of the integrated PL intensity for the InGaN epifilm sample. The temperature dependence obeys the relation $I_{P L} \propto\left[1+A \exp \left(T / T_{0}\right)\right]^{-1}$.

scattering [15] and localization of an exciton [16]. APF also provides a model system for the study of carrier transport in percolative solids [17] as well as a simple disordered system for the study of vibrational properties [18].

Another piece of evidence to support the existence of APF is the temperature dependence of the PL peak energy as shown in figure 3 . It shows an anomalous behaviour, in which the peak energy decreases, then increases with increasing temperature in the range $40-100 \mathrm{~K}$ and finally decreases with increasing temperature. This anomalous behaviour, called the inverted S-shape, has also been observed in disordered superlattices [11] and other ternary III-V semiconductor alloys [19]. Note that, in those studies, the PL intensities as a function of temperature can also be fitted well by equation (1). This result has been attributed to the effect of localized states caused by potential fluctuations $[10,11,19]$. Thus, the recombination of electron-hole pairs is considered to occur around the localized states. It is expected that they have localized states with different extents of localization owing the nature of random alloy fluctuations. At low temperature, carriers can recombine at energy states with local potential minima. As temperature slightly increases, weakly localized carriers are thermally excited and would either recombine nonradiatively or redistribute to other strongly localized states. Thus, the PL peak energy decreases with increasing temperature. After the effect of redistribution is saturated, the thermal energy can excite carriers to higher localized states, and hence the PL peak energy increases. As the 


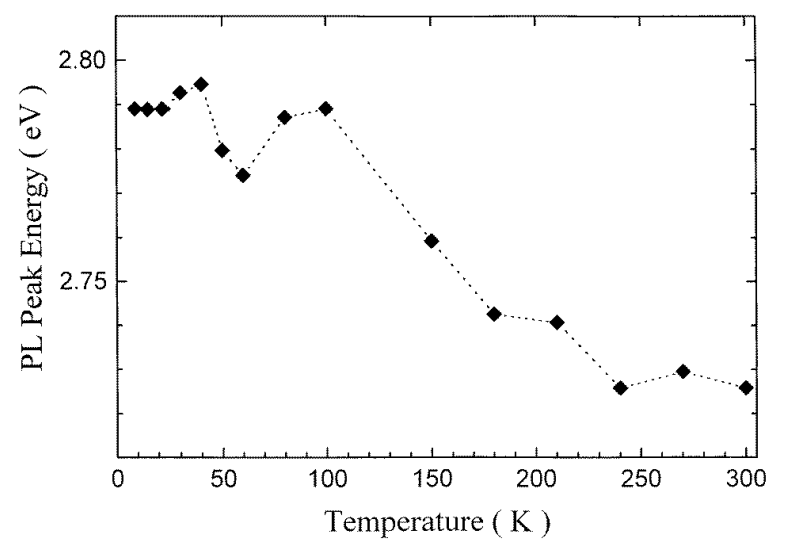

Figure 3. Temperature dependence of the PL energy for the InGaN epifilm sample.

temperature increases further, a red shift evolves. This means that the contribution to the emission process in part under the effect of strong localization of particles becomes smaller than the temperature-induced bandgap shrinkage, and the temperature dependence of the emission energy follows the thermal property of the bandgap which decreases with increasing temperature.

Additional evidence for the existence of the localized states due to APF of the random alloy is provided by the measurement of the excitation intensity dependence of the PL spectra as shown in figure 4. It is seen that the PL peak energy increases with increasing excitation intensity. This peculiar result can also be understood in terms of the localized states due to alloy fluctuations. Because the localized states located at the band edge are limited in number, they can be easily occupied by the photoexcited carriers. Increasing the excitation intensity will increase the state filling, and hence the emission energy is also increased. Therefore, all our PL measurements can be explained by APF in a consistent way. We may suggest that compositional fluctuations do exist in InGaN epilayers.

In order to have further evidence to support the existence of APF, we performed the PC measurements. Figure 5 shows a typical PC spectrum of the undoped InGaN sample at $10 \mathrm{~K}$. The PC exhibits a broad spectrum centred at $2.95 \mathrm{eV}$ with a small hump around $3.45 \mathrm{eV}$ corresponding to the absorption of the GaN buffer layer. The spectrum is flat at the lower energies indicating negligible deep states. Also, this result indicates a Stokes shift between the PL peak position and the band edge transition energy in this InGaN sample. The inset of figure 5 shows the shift of the optical transition energy from the temperature evolution of the PC spectra for this InGaN sample. The full line in the figure represents the best fit to the Varshni empirical equation [20]

$$
E(T)=E_{0}(0)-\alpha T^{2} /(\beta+T)
$$

where $E_{0}(0)$ is the transition energy at $0 \mathrm{~K}$ and $\alpha$ and $\beta$ are the Varshni thermal coefficients. The parameters obtained from the best fit for the sample are given in the figure which are reasonably comparable with the results of an undoped $\mathrm{In}_{0.14} \mathrm{Ga}_{0.86} \mathrm{~N}$ sample obtained by photoreflectance (PR) spectroscopy [21]. Within the temperature range

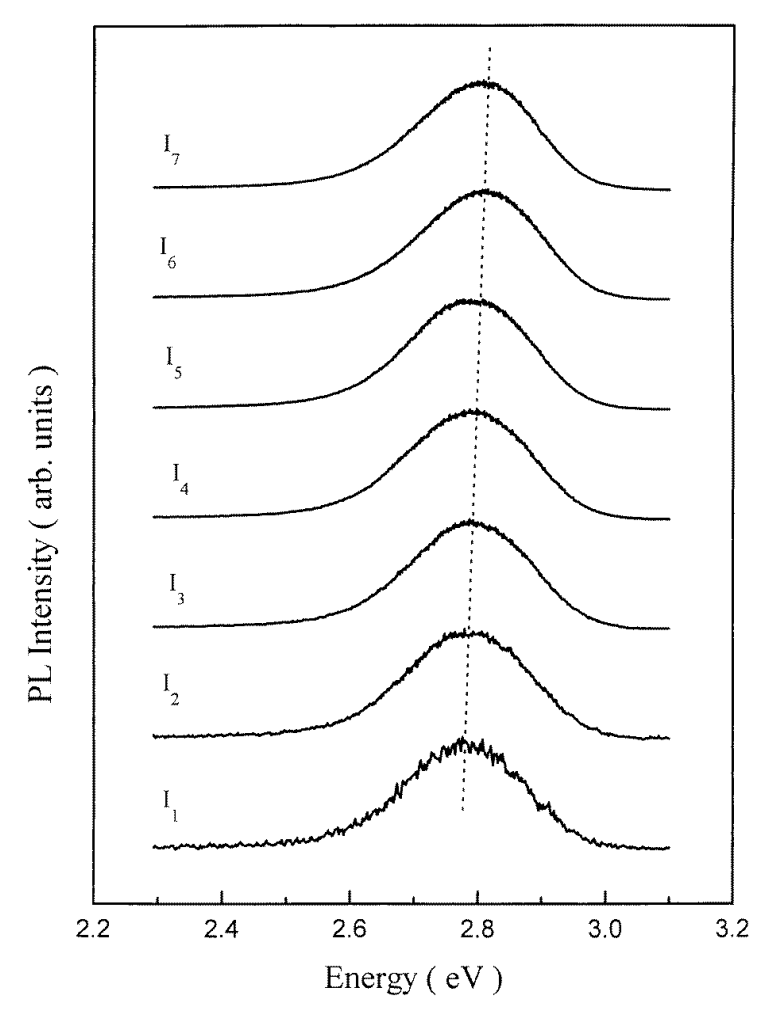

Figure 4. Excitation intensity dependence of the PL spectrum taken at $10 \mathrm{~K}$. The PL energy increases with increasing excitation intensity, $I$, where $I_{1}, I_{2}, I_{3}, I_{4}, I_{5}, I_{6}$ and $I_{7}$ are 0.004, 0.035, $0.12,0.26,0.28,0.3$ and $0.4 \mathrm{~W} \mathrm{~cm}^{-2}$ respectively.

studied in this work, the peak energy of PC spectra is larger than that of the PL spectra shown in figure 3 by a value of $165 \pm 5 \mathrm{meV}$. The apparent difference in the peak energy between PL and PC spectra can be attributed to the APF effect and will be discussed later. A more surprising feature of the photoresponse in the InGaN films is the observation of the PPC effect as shown in figure 6. We can see that the conductivity increases gradually after the sample is exposed to light and that the light-induced conductivity can persist for a very long period of time after the light is removed, which can extend to more than $10^{3} \mathrm{~s}$ at RT. A similar PPC effect has been observed in many important semiconductor thin films and heterostructures [7, 22-24]. Here, we report the observation of the PPC effect in InGaN epifilms for the first time. Our observed PPC effect can be easily understood in terms of APF. At low temperature, the photoexcited electrons and holes can be trapped and spatially separated by potential fluctuations, the recombination of electron-hole pairs is inhibited, and hence PPC occurs.

The kinetics of PPC relaxation shows a stretchedexponential behaviour which can be expressed as

$$
I_{P P C}(t)=I_{P P C}(0) \exp \left[-(t / \tau)^{\beta}\right]
$$

where $I_{P P C}(0)$ is the PPC buildup level at the moment of light excitation being removed, $\tau$ is the PPC decay time constant and $\beta$ is the decay exponent. Figure 7 shows a representative plot of $\ln \left[\ln I_{P P C}(0)-\ln I_{P P C}(t)\right]$ versus $\ln (t)$. The good linear behaviour of the plot demonstrates that the PPC decay is well described by equation (3). 


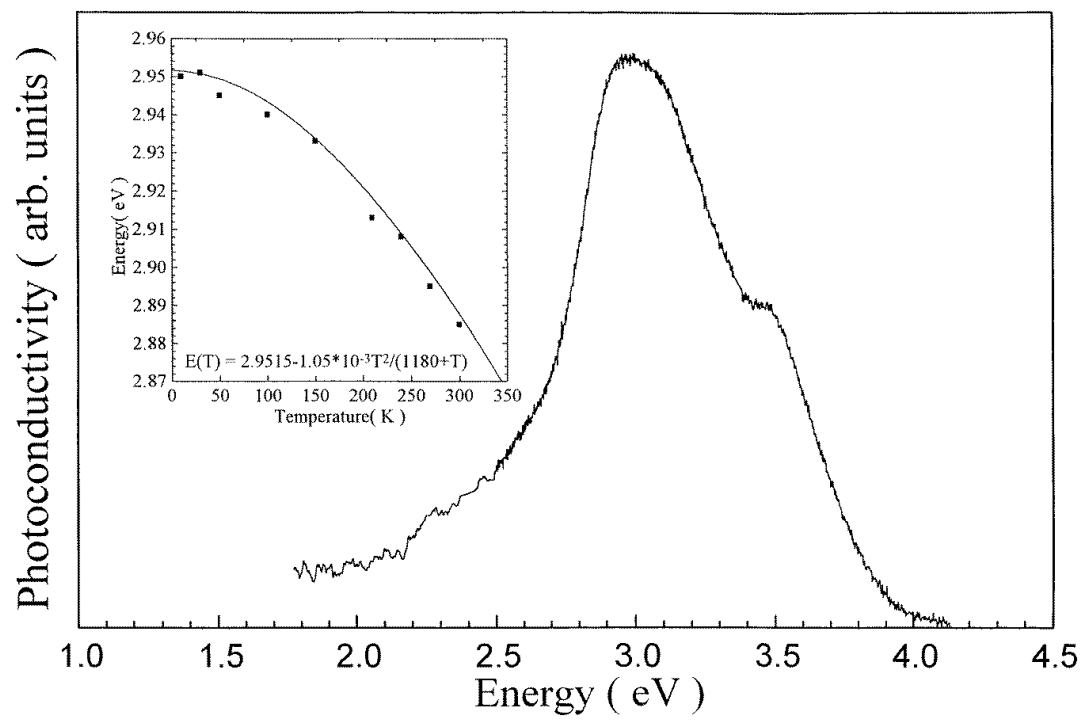

Figure 5. PC spectrum of an undoped InGaN sample taken at $10 \mathrm{~K}$. The inset shows the temperature dependence of the optical transition energy obtained from the PC spectra of the sample.

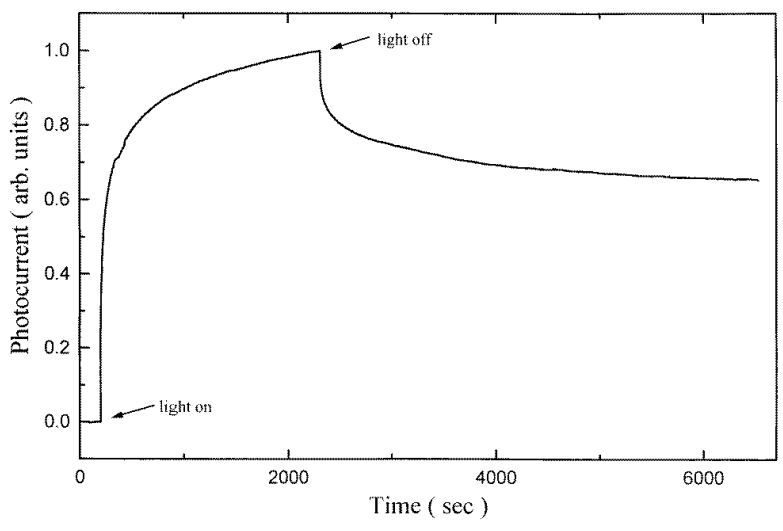

Figure 6. Typical buildup and decay behaviour of PPC in the undoped $\mathrm{In}_{0.30} \mathrm{Ga}_{0.70} \mathrm{~N}$ film at $10 \mathrm{~K}$.

The stretched-exponential relaxation has been commonly observed in disordered systems $[7,25,26]$. The origin of the stretched-exponential relaxation can be understood by the fact that the rate constant $v$ of a physical quantity $\Delta$ in the decay-rate equation is time dependent, that is

$$
\mathrm{d} \Delta / \mathrm{d} t=-v(t) \Delta .
$$

This equation describes the decay rate for small departures of $\Delta$ from equilibrium. A single-value rate constant $v$ will yield the conventional Debye relaxation. Many previous investigations [27-29] showed that if the density of states is exponentially distributed in terms of energy, such as the bandtail states in disordered semiconductors, the rate constant $v$ exhibits a power-law time dependence. Inserting a powerlaw time dependence for $v$ into equation (4) and integrating immediately yields a stretched-exponential function. Thus, the stretched-exponential decay reveals similarities of the present system to disordered systems. This implies that the origin of the localized band-tail states in InGaN films is due to the disorder behaviour of alloy compositional fluctuations.
We therefore can see that APF can also be used to interpret the observed PPC effect quite well.

From the temperature evolution of the PPC effect, the carrier capture barrier $\Delta E$, which characterizes the energy difference between localized states and the band edge, can be obtained from the temperature dependence of $\tau$ :

$$
\tau=\tau_{0} \exp (\Delta E / k T)
$$

It was found that $\Delta E$ is about $157 \mathrm{meV}$ as shown in the inset of figure 7 , which is very close in value to that of the difference in the peak energy between PL and PC spectra mentioned above. Following the existence of localized states introduced by alloy disorder, carrier transitions for luminescence occur mostly between localized states in the tails of conduction and valence bands, while the PC peak energy reflects the bandgap absorption. Thus, the discrepancy in the peak energy between PL and PC spectra characterizes the depth of localized states from the band edge, which is expected to be the same as the carrier capture barrier $\Delta E$ obtained from the PPC decay kinetics. This prediction is indeed consistent with our experimental measurement. Thus, our result unambiguously implies that the APF effect leads to the existence of PPC in InGaN epilayers.

A further indication of strong localization effects is obtained from the investigation of the undoped $\operatorname{~n}_{x} \mathrm{Ga}_{1-x} \mathrm{~N}$ epifilms with varying indium components, $x$. Figure 8 shows the measured PL and PC spectra of an undoped $\operatorname{In}_{0.15} \mathrm{Ga}_{0.85} \mathrm{~N}$ sample at $10 \mathrm{~K}$. The PL spectrum is dominated by an emission band with a peak centred at $3.06 \mathrm{eV}$ while the PC spectrum exhibits a broad spectrum showing the absorption edge of the InGaN layer at $3.17 \mathrm{eV}$. The localization depth of this sample is found to be $110 \mathrm{meV}$ which is smaller than that (165 meV) of the sample with a larger indium component discussed above. Recently, Shan et al have performed measurements of PL and PR in $\operatorname{In}_{x} \mathrm{Ga}_{1-x} \mathrm{~N}$ alloys $(0<x<$ 0.2) [21]. They found a large Stokes shift between the PL peak position and the band edge transition energy, which also decreases with decreasing indium content. The decrease 


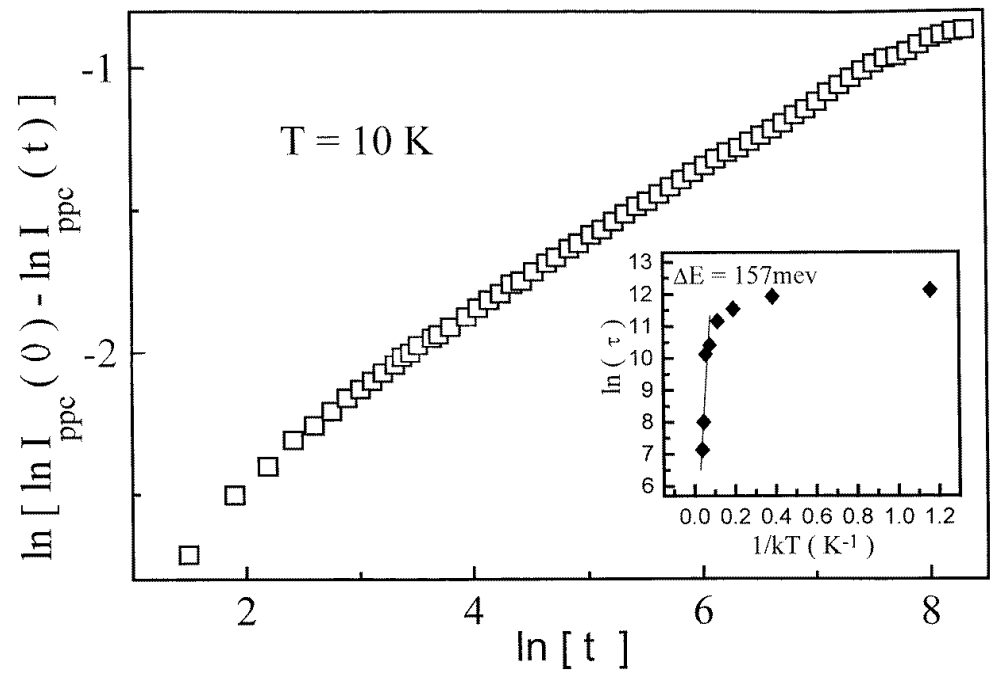

Figure 7. Plot of $\ln \left[\ln I_{P P C}(0)-\ln I_{P P C}(t)\right]$ versus $\ln (t)$ for the undoped $\operatorname{In}_{0.30} \mathrm{Ga}_{0.70} \mathrm{~N}$ film at $10 \mathrm{~K}$. The linear curve indicates that the PPC decays according to the stretched-exponential function $I_{P P C}(t)=I_{P P C}(0) \exp \left[-(t / \tau)^{\beta}\right]$. The inset shows the carrier capture barrier $\Delta E$ obtained according to $\tau=\tau_{0} \exp (\Delta E / k T)$.

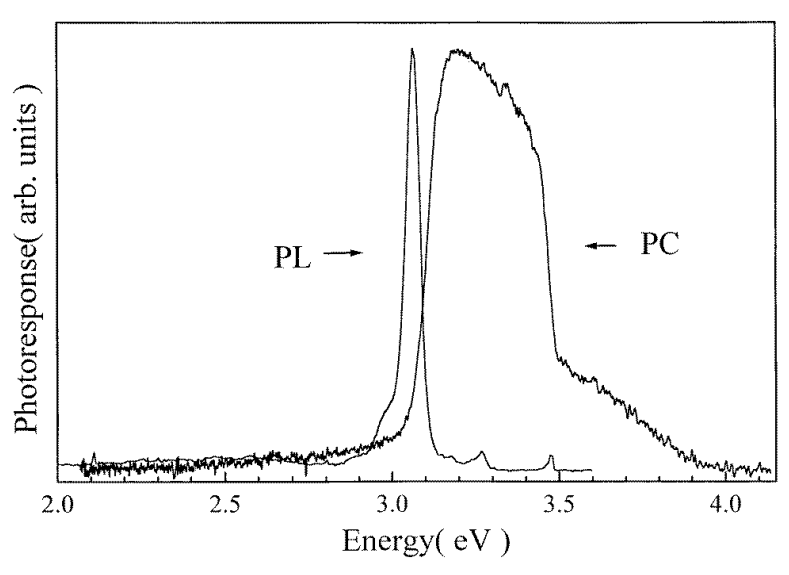

Figure 8. PL and PC spectra of an undoped $\operatorname{In}_{0.15} \mathrm{Ga}_{0.85} \mathrm{~N}$ epifilm sample taken at $10 \mathrm{~K}$.

in the depth of localized states from the band edge for the alloys with small indium components is also consistent with the APF effect. Therefore, our results clearly indicate the existence of compositional fluctuations in the $\operatorname{In}_{x} \mathrm{Ga}_{1-x} \mathrm{~N}$ epifilms.

\section{Conclusion}

In summary, the PL and PC behaviours have been investigated in InGaN alloy epilayers. Several striking features of the optical properties have been observed, which include temperature and excitation intensity dependence of the PL intensity and peak position, the existence of a large Stokes shift between the PL peak position and the band edge transition energy and the observation of PPC in InGaN layers. All these phenomena can be well interpreted by APF in a consistent way. Thus, our results strongly suggest that compositional fluctuations do exist in InGaN epilayers. We point out that APF may be the underlying mechanism responsible for the widely observed localized excitons, which are now considered to play an important role in the emission of InGaN-based devices.

\section{Acknowledgment}

This work was partially supported by the National Science Council of the Republic of China.

\section{References}

[1] Chichibu S, Azuhata T, Sota T and Nakamura S 1997 Mater. Res. Soc. Symp. Proc. 499653

[2] Narukawa Y, Kawakami Y, Funato M, Fujita S, Fujita S and Nakamura S 1997 Appl. Phys. Lett. 70981

[3] Smith M, Chen G D, Lin J Y, Jiang H X, Asif Khan M and Chen Q 1996 Appl. Phys. Lett. 692837

[4] Harris C I, Monemar B, Amano H and Akasaki I 1995 Appl. Phys. Lett. 67840

[5] Chichibu S, Azuhata T, Sota T and Nakamura S 1996 Appl. Phys. Lett. 694188

[6] Narukawa Y, Kawakami Y, Fujita S, Fujita S and Nakamura S 1997 Phys. Rev. B 55 R1938

[7] Chu L H, Chen Y F, Chang D C and Chang C Y 1995 J. Phys.: Condens. Matter 74525

[8] Chen Y F, Lin L Y, Shen J L and Liu D W 1992 Phys. Rev. B 4612433

[9] Van der Stricht W, Moerman I, Demeester P, Considine L, Thrush E J and Crawley J A 1997 MRS Internet J. Nitride Semicond. Res. 216

[10] Street R A, Searle T M and Augustein I G 1974 Amorphous and Liquid Semiconductors ed J Stuke and W Brenig (London: Taylor and Francis) p 953

[11] Yamamoto T, Kasu M, Noda S and Sasaki A 1990 J. Appl. Phys. 685318

[12] Van de Walle C G and Martin R M 1986 Phys. Rev. B 345621

[13] Blood P and Grassie A D C 1984 J. Appl. Phys. 561866

[14] Singh J and Bajaj K K 1986 Appl. Phys. Lett. 481077

[15] Parayantha P and Pollak F H 1984 Phys. Rev. Lett. 521822

[16] Lai S and Klein M V 1980 Phys. Rev. Lett. 441087

[17] Jiang H X and Lin J Y 1990 Phys. Rev. Lett. 642547

[18] de Gironcoli S and Baroni S 1992 Phys. Rev. Lett. 691959 
[19] Olshoorn S M, Driessen F A J M, Eijkelenboom A P A M and Giling L J 1993 J. Appl. Phys. 737798

[20] Varshni Y P 1967 Physica 34149

[21] Shan W, Little B D, Song J J, Feng Z C, Schurman M and Stall R A 1996 Appl. Phys. Lett. 693315

[22] Lang D V and Logan R A 1977 Phys. Rev. Lett. 39635

[23] Mooney P M 1990 J. Appl. Phys. 67 R1

[24] Jiang H X and Lin J Y 1990 Phys. Rev. Lett. 57873
[25] Kakalios J, Street R A and Jackson W B 1987 Phys. Rev. Lett. 591037

[26] Chen Y F, Huang S F and Chen W S 1991 Phys. Rev. B 44 12748

[27] Campos M, Giacometti J A and Silver M 1979 Appl. Phys. Lett. 34226

[28] Jackson W B 1988 Phys. Rev. B 383595

[29] Crandall R S 1991 Phys. Rev. B 434057 